\title{
Safety-Active Catheter with Multiple-Segments Driven by Micro-hydraulic Actuators
}

\author{
Koji Ikuta, Hironobu Ichikawa, and Katsuya Suzuki \\ Nagoya University, Graduate School of Engineering, \\ Department of Micro System Engineering, \\ Furo-cho, Chikusa-ku, Nagoya, Aichi, 464-8603, Japan \\ Tel: +81 52-789-5024, Fax: +81 52-789-5027 \\ ikuta@mech.nagoya-u.ac.jp
}

\begin{abstract}
We proposed and developed an innovative active catheter with multisegments that can bend in the narrow blood vessel. Micro hydraulic actuator system based on new principle has been developed by the authors. Moreover, new micro fabrication method named hybrid stereolithograpy (IH process) requiring any assemble process is introduced for leakage-free packaging catheter. Total system with pressure control system was made. Good safety and drive performance were verified experimentally. We also devised theoretical models of these valves to facilitate quantitative design and to extend applications for safe medical tools.
\end{abstract}

\section{Introduction}

Non-invasive or minimally invasive examinations and medical treatments are increasing in number as society ages. Catheterization is a typical minimally invasive medical technique. A catheter is a thin tube which is inserted into a peripheral vessel in an upper or lower limb for diagnosis or treatment of vascular system diseases; it is mainly used to measure internal pressure of the cardiovascular system, collect blood samples and inject contract medium for angiography. Catheterization is popular in clinical surgery because it allows minimally invasive diagnosis.

However, it is very difficult to insert a catheter with a guide wire which is now widely used, due to not only small inner diameter but also bending, twisting and frequent branching of vessels. Therefore, there is a need to develop an active catheter which can bend and twist its shaft in any direction and choose a direction at a branch point of a vessel [1][2][3]. These studies attached electric actuators on the parts to be inserted into the body, such as SMA or a special polymer that requires a high electric current of several amperes; this could be dangerous, with a risk of electric leakage in case of an accident such as breakage. There are also other problems that prevent practical application such as poor durability due to the complex internal mechanism of a catheter, and increasing lines associated with increasing joints [4].

To solve these problems, this study employed normal physiological salt solution for both drive and drive signal transmitter, and proposed a concept of a novel, safetyactive catheter with simple mechanism using a hydraulic actuator. A prototype of a new type of microvalve, which is the key to this catheter, was developed and examined. The valve was then miniaturized to fit into an actual catheter. The control system was developed and safe actuation in the model blood vessel was verified. 


\section{Proposal of Hydraulic Active Catheter}

Figure 1 shows the concept of the hydraulic active catheter proposed in this study. The features of this system are as follows:

1) Electricity is not used on any part to be inserted into the body.

2) The drive fluid also works as a signal transmitter.

3) The number of diving lines will not increase with the number of active joints due to the single drive system.

In summary, this system consists of bellows as an actuator at each joint and a drive tube to control the bellows as shown in the figure.

Each joint is designed to bend while a bellows, as an actuator, is extended by fluid supply. Since normal saline is used as the drive fluid, safety is ensured because only harmless normal saline, instead of an electric leak, would leak if the catheter were to break inside the body.

The problem is how to achieve compact wiring in designing micromachinery. If a signal line is required for each new joint, reliability is impaired due to complicated signal lines at the proximal end, especially in the case of a long, thin system like a catheter. Our proposed single drive system allows a multi-joint design, free from complicated signal lines.

\section{Band Pass Valve (BPV)}

In this study, we developed a so-called "Single-input, multi-output" control mechanism which uses drive fluid that can also act as a signal transmitter and which controls each bellows (valve) independently through the drive fluid. We invented a static pressure Band Pass Valve (BPV) for independent active drive to ensure a simple mechanism and ease of control.

The concept of the BPV drive is shown in Fig. 2. This valve consists of a pair of valves: a High Pass Valve (HPV) and a Low Pass Valve (LPV). When the inner pressure of the tube (a drive system) reaches specific pressure P1, the lower valve (HPV) opens to make BPV open, and therefore drive fluid is supplied to the bellows (a drive actuator). When the inner pressure increases to $\mathrm{P} 2$, the upper valve (LPV) shuts to make BPV shut, and therefore the mechanism stops supplying fluid to the bellows.

Through these actions, BPV remains open within the preset specified pressure range only (from $\mathrm{P} 1$ to $\mathrm{P} 2$ of inner tube pressure in the figure) and $\mathrm{BPV}$ supplies drive fluid into the bellows.

Figure 3 shows the general scheme of the single-input, multi-output control mechanism of multi-joint active catheter using this BPV. The application of three joints is shown in this figure. A set of bellows (an actuator) and BPV corresponds to a joint, and they are arranged parallel to the drive tube. The drive pressure range of BPV on each joint is set to be different from each other.

By increasing the inner pressure of the drive tube stepwise to the activation pressure band of each valve, only the target valve opens and fluid is discharged each time. Therefore, only the target bellows connected to the valve is supplied with fluid and extended, and only the target joint will bend. 


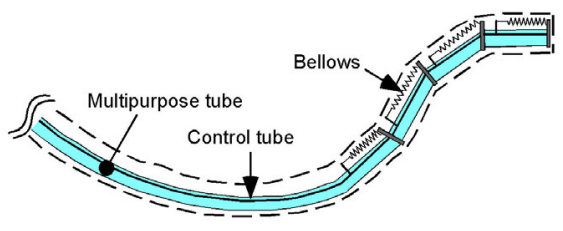

Fig. 1. Basic concept of Micro Hydraulic Active Catheter with Multi-degrees of Freedom

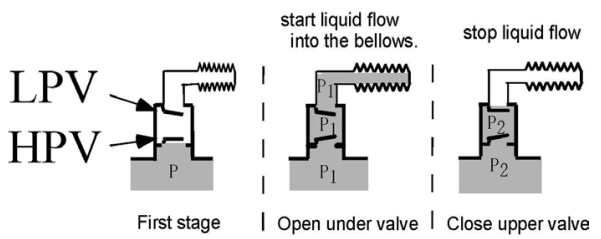

Fig. 2. Basic Principle of Band Pass Valve

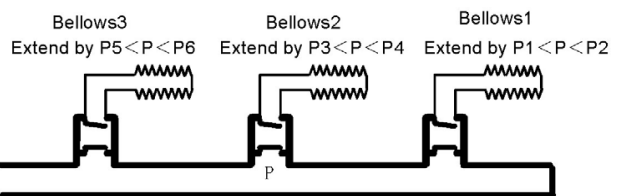

By setting valve $\mathrm{P} 1<\mathrm{P} 2<\mathrm{P} 3<\mathrm{P} 4<\mathrm{P} 5<\mathrm{P} 6 \cdots$ each bellows can be independently controlled
Fig. 3. The one input multi-output control mechanism

In this way, any valve state can be controlled by regulating the inner pressure of one drive tube using the Band Pass Valve (BPV), and so each associated joint can be controlled.

\section{Design and Development of a Prototype Band Pass Valve (BPV)}

To configure the proposed BPV, it is necessary to develop a pair of valves as mentioned above, HPV and LPV.

\subsection{Design of HPV}

Figure 4 shows the basic structure of the High Pass Valve (HPV). This valve is structured to block the flow path with a core material which is pressed by an elastic membrane.

No leakage occurs from HPV in its closed state due to the complete blocking of the flow path with the core material. When the inner pressure exceeds the elastic membrane pressure against the core material, the valve opens as the core material is lifted, and fluid is discharged.

\subsection{Design of LPV}

Figure 5 shows the basic structure of the Low Pass Valve (LPV). This valve is structured such that its elastic membrane covers a keyhole shaped channel.

When the inner pressure is low, fluid discharges from the gap between the flow path and the elastic membrane. When the inner pressure increases, the elastic membrane is pressed to block the flow path and thus fluid discharge is stopped. 


\subsection{Development of a Prototype Band Pass Valve (BPV)}

Prototypes of HPV and LPV were developed as to verify the mechanism based on the valve designs described in sections 4.1 and 4.2 . Then, by connecting these two prototype valves, a prototype BPV was made as shown in Fig. 6.

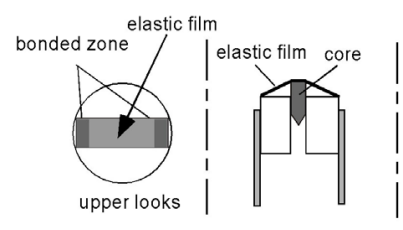

Fig. 4. Basic structure of HPV

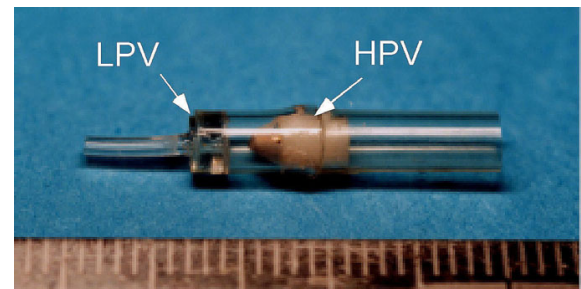

Fig. 6. Prototype of BPV

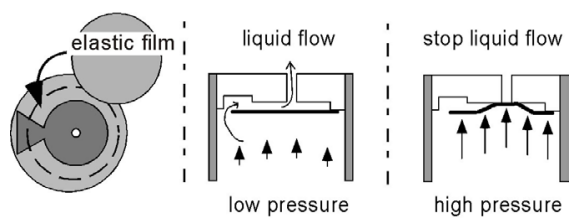

Fig. 5. Basic structure of LPV

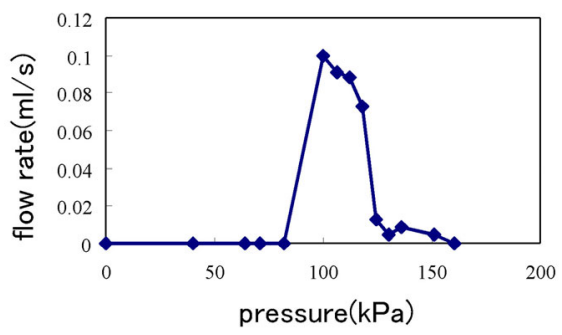

Fig. 7. Experimental evaluation on Pressure dependent flow rate of BPV

\subsection{Characteristic Evaluation of BPV}

The characteristics of the prototype BPV were evaluated. Figure 7 shows the test results of the relationship between the inner pressure and the outflow discharge of the prototype BPV. These data verified showed that in the band pass drive, fluid passed through the valve only when a specific pressure was applied.

\section{Construction of Theoretical Model for Quantitative Design}

Theoretical models of two component valves of BPV, the LPV and HPV, were constructed in order to design the BPV quantitatively. In addition, an experiment to verify the constructed model was conducted.

\subsection{Theoretical Model of LPV}

First, the theoretical model of LPV was developed as shown in Fig. 8. Bernoulli's theorem was applied to this model to derive Eq. (1). Let $\mathrm{k}$ represent the modulus of elasticity of a circular elastic membrane of $1 \mathrm{~mm}$ diameter. This equation states that the pressure difference to close the valve ( $\left.\mathrm{P}_{\text {close }}\right)$ is inversely proportional to the cube of the diameter $\mathrm{d}$ of the elastic membrane. 


\subsection{Theoretical Model of HPV}

Next, the theoretical model of HPV was developed as shown in Fig. 9. Equation (2) was derived by considering the force balance on the valve. ( $\mathrm{F}$ is a bias force applied to core from elastic film.)

This equation states that the pressure difference to open the valve $\left(\mathrm{P}_{\text {open }}\right)$ is directly proportional to the modulus of elasticity of the membrane.

$$
\Delta P_{\text {close }}=\frac{64 h^{3} k}{d_{0}^{2} \pi} \cdot \frac{1}{d^{3}} \quad \text { (1) } \quad \Delta P_{\text {open }}=\frac{k \cdot\left(l+l_{0}\right)+F}{A}
$$

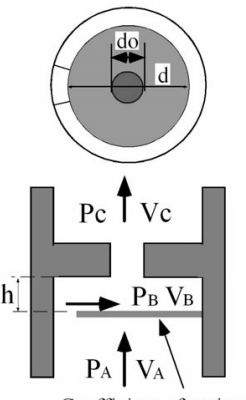

Coefficient of spring $k$

Fig. 8. Theoretical model of LPV

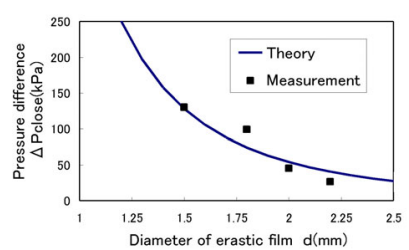

Coefficient of spring $\mathrm{k}$

elongation from the natural length 1

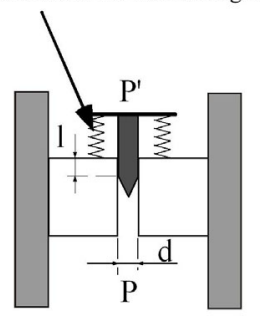

Fig. 9. Theoretical model of LPV

Fig. 10. The comparison of experimental result and theoretical model
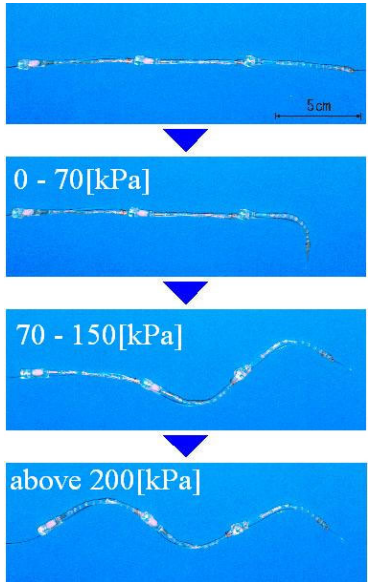

Fig. 11. First prototype of an active catheter with four active segments for verification of basic principle

\subsection{Verification Experiment of Basic Principle}

An experiment was conducted using a prototype LPV to verify our theoretical model. Measurement data were collected on the relationship between the diameter of the elastic membrane which blocks the flow path and the pressure difference when the valve is closed in this experiment. The results are shown in Fig. 10. The chart indicates that the data are very close to the ideal curve, and hence this theoretical formula enables the LPV to be quantitatively designed to close at a given pressure.

However, the theoretical model of the HPV could not be verified because of difficulty of quantitatively measuring the modulus of elasticity of the membrane. Nevertheless, the quantitative design with the developed model is sufficiently practical owing to the very simple operation mechanism of the HPV. 


\section{Driving Experiment of the First Prototype}

A prototype of a hydraulic active catheter was developed which has three joints with BPV of the prototype verification model. A driving experiment was conducted with this catheter on a single-input, multi-output control mechanism (Fig. 11).

The results confirmed that each joint was driven independently by the pressure control on one drive tube, verifying that a single-input, multi-output control mechanism using drive fluid as a signal transmitter has been achieved.

\section{Miniaturization of Band Pass Valve}

The driving experiments proved the usefulness of the Band Pass Valve (BPV). However, the maximum diameter of the prototype active catheter used in the driving experiments was $6 \mathrm{~mm}$, which is sufficient for experiments but too large for catheter use, and therefore it should be made smaller. To do this, the size of the valve must be reduced.

\subsection{Problems for Miniaturization}

Generally, miniaturization involves the following problems:

1) Assembly operation

2) Bonding part

In this system, each part should be made smaller to reduce the size of the valve. However, it is difficult to assemble such micro parts, and furthermore, the bonding part for valves hinders miniaturization. Airtightness is another important issue as the fluid must not leak from the valve.

To solve these problems, we devised the Hybrid IH process [5] .

\subsection{New Fabrication Method Using Hybrid Micro-stereolithography (IH Process)}

The Hybrid IH process is a shaping method developed in our laboratory, by the process shown in Fig.12. In this shaping method, first, UV curable epoxy resin is cured by laser and laminated as in the usual shaping method. Next, a part made from a different material is loaded in the middle of the shaping process, and a single piece is formed.

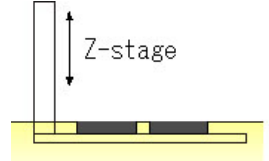

(1)

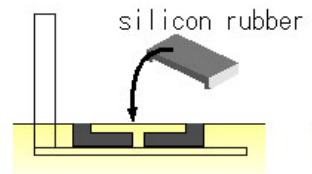

(2)

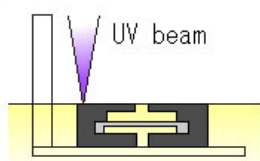

(3)
Fig. 12. Fabrication process of Hybrid micro stereolithography $\quad(\mathrm{IH}$ Process)

The features of the Hybrid IH process are as follows:

1) No need for assembly operation and bonding

2) High airtightness

3) 3D structures can be produced. 
A leak-free miniature 3D structure can thus be easily produced by the Hybrid $\mathrm{IH}$ process.

\subsection{Design of Micro-HPV and Micro-LPV}

The following factors were taken into consideration to produce microvalves by the Hybrid IH process.

1) Selection of desirable materials for Hybrid IH process

2) Appropriate design for a single-piece style

Considering these points, the LPV and HPV are designed to make the most of the characteristics of the Hybrid IH process.

The LPV in the verification model had a keyhole shaped flow path covered with the circular elastic membrane. The shapes of the flow path and the elastic membrane were modified for miniaturization as shown in Fig. 13. The material of the elastic membrane was replaced with silicone rubber and formed into a platy, legged shape. There is no need for a bonding surface because the part is held in an insertion structure. The drive mechanism is the same as that in the verification model: when the inner pressure increases, the silicone rubber part changes shape, plugs the fluid path and stops the fluid discharge.

As for the HPV, the core material and the elastic membrane blocked the flow path in the verification model. Therefore it consisted of many parts and also the assembly was complex. These parts were therefore integrated into one silicone rubber part in the miniaturized model (Fig. 14); again, no bonding part is needed because the silicone rubber part is held in an insertion structure. The drive mechanism is also the same as that in the verification model. No leakage occurs in the closed state due to the complete blocking of the flow path. When the inner pressure increases, fluid is discharged from a gap which is formed by distortion of the silicone rubber part.

Since the mounting location on the drive tube is taken into consideration in designing both valves, the whole system is sleek even after mounting the valves to the tube.

\subsection{Fabrication of a Prototype Micro-BPV}

Miniaturized prototypes of the LPV and HPV having the designs described above were combined to make the BPV. Figure 15 shows a comparison of a micro-BPV made by the Hybrid $\mathrm{IH}$ process and a BPV unit used as a verification model in the driving experiment.

The valve diameter is reduced from $6 \mathrm{~mm}$ in the verification model to $3 \mathrm{~mm}$ using the Hybrid IH process as the figure shows.

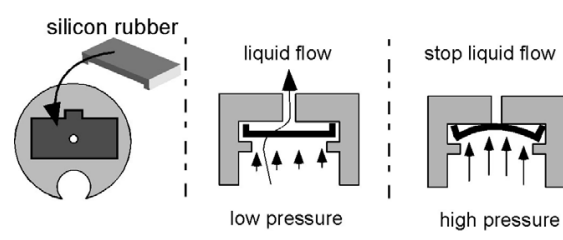

Fig. 13. Design of micro LPV
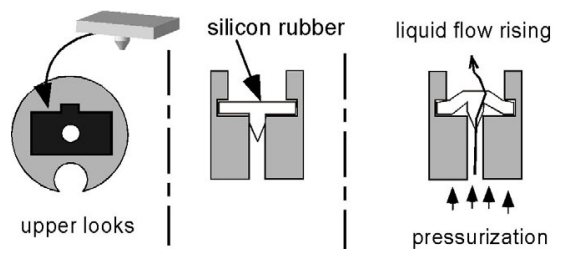

Fig. 14. Design of micro HPV 

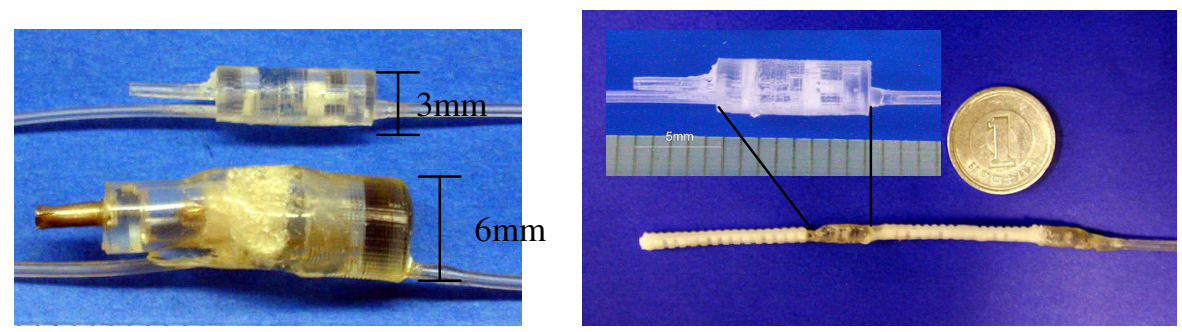

Fig. 15. Prototype of BPV and miniaturized BPV in $3 \mathrm{~mm}$ diameter

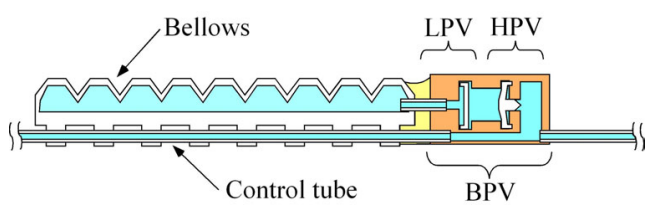

Fig. 16. Cross section of an one segment of active catheter

\section{Prototyping Two-Segments Active Catheter}

A two-segments active catheter was constructed using the developed micro-BPV. The bellows and BPV are arranged along the drive tube. The size of a joint is $40 \mathrm{~mm}$ in length and $3 \mathrm{~mm}$ in diameter. The bellows as an actuator is made from silicone rubber; it extends easily on one side but not on the other, so supplied fluid readily bends it.

Figure 17 shows an prototyped active catheter on the finger tip. Each segment can be bent independently as shown in this figure. Master-slave pressure control system shown in Figure 18 was made for simple control by the doctors. Pressure range is enough low so as to decrease risk of break.

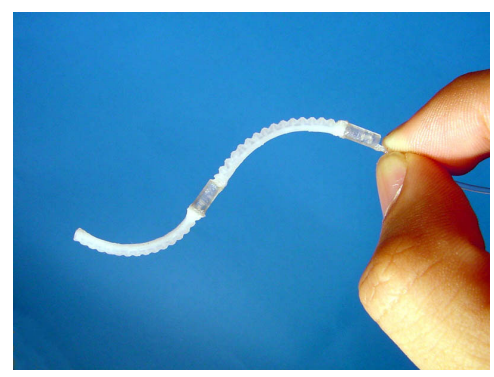

Fig. 17. Miniaturized hydraulic active catheter with two segments

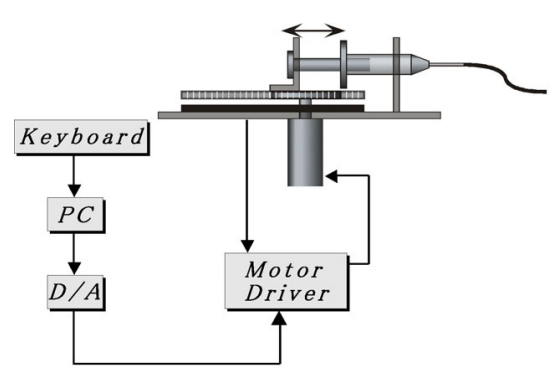

Fig. 18. Pressure control system for masterslave bending of each segment

\section{Insertion Experiment}

An insertion experiment was performed using the constructed active catheter with two segments 
The pathway for this insertion experiment is $5 \mathrm{~mm}$ in width and $5 \mathrm{~mm}$ in depth. Figure 19 shows a running insertion experiment, confirming that the two-segments active catheter is inserted smoothly. Moreover, it can easily be inserted into places where one-joint catheters are difficult to insert. In another experiment in the branched blood vessel, the active bending ability of this tool was verified as shown in Fig.20. It was much easy to select direction at the branching point.

Even a 3-mm diameter catheter can be used if the sites are confined. For future application to thin blood vessels such as capillaries, the system needs to be made even smaller.

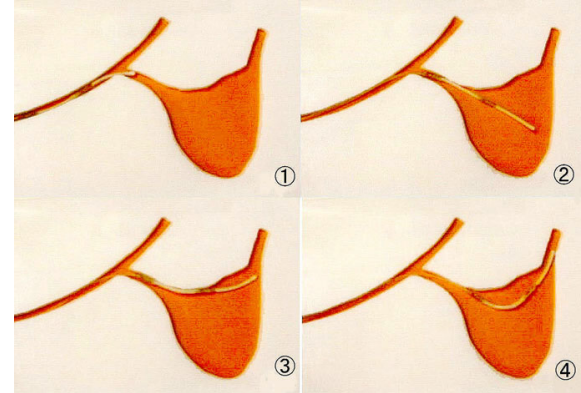

Fig. 19. Insertion experiment into blood vessel model
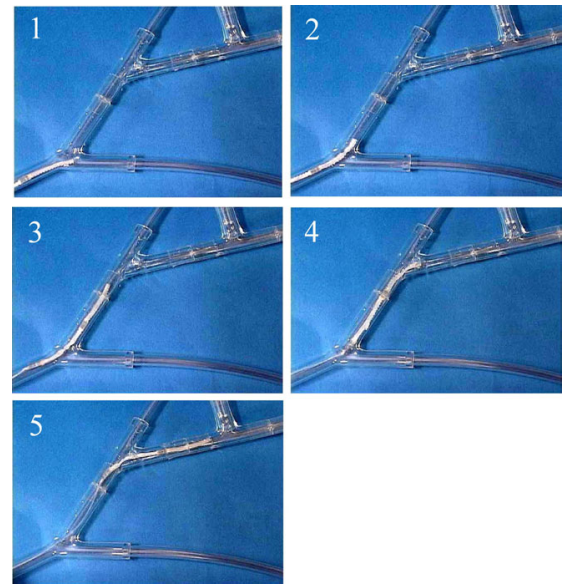

Fig. 20. Selecting direction at in the branched blood vessel model

\section{Conclusion}

An innovative hydraulic micro actuator system that controls multiple segments independently with single-input signal through fluid as a signal transmitter for safe medical tools was developed. And the prototype hydraulic active catheter using this mechanism was developed successfully. A driving experiment was conducted with the prototype active catheter and the results were favorable. Verification experiments of the theoretical models of valves confirmed that quantitative design was made. Moreover, a valve unit having the same function was made smaller using the Hybrid micro streolithography (IH process), involving micromachining technology developed by the authors, and an actual-size two-segment active catheter was constructed with these valves. The usefulness and safety feature of the developed active catheter was demonstrated by an insertion experiment.

\section{References}

1. Shuxiang Guo, Toshio Fukuda, Fumihito Arai, Makoto Negoro, Keisuke Oguro, A Study on Active Catheter System Structure Experimental Results and Characteristic Evaluation of Active Catheter with Multi DOF, Journal of the Robotics Society of Japan, Vol.14 No.6, pp.820-835, 1996. 
2. M.Tanimoto et al, Improvement of Safety in Telemedicime System, Proc. of 3rd Robotics Symposia, pp.139-144,1998.

3. M.Esashi,Moption Control System by Micromachining, Plenary Proc. of 1998 JSME Conference on Robotics and Mechatronics (ROBOMEC 98), pp.5-13, 1998.

4. K.Ikuta, M.Nokata, Minimum Wire Drive of Multi Micro Actuators Journal of the Robotics Society of Japan, Vol.16, No.6, pp.791-797, 1998.

5. K. Ikuta, S. Maruo, T. Fujisawa and A. Yamada, "Micro Concentrator with Opto-sense Micro Reactor for Biochemical IC Chip Family - 3D Composite Structure and Experimental Verification -," Proceedings of the IEEE International Workshop on Micro Electro Mechanical Systems(MEMS99), 376-381, 1999. 